\title{
Development and Evaluation of Inexpensive Ultrasound Using A-Mode and M-Mode Signals to Identify Lung Depth and Avoid Risk of Pneumothorax in Acupuncture
}

\author{
Ying-Ling Chen ${ }^{1} \cdot$ Mark C. Hou ${ }^{2}$ (D) Shun-Chang Chang ${ }^{2} \cdot$ Kai-Wen Chuang $^{2} \cdot$ Po-Yang Lee $^{3} \cdot$ Chih-Chung Huang $^{3}$
}

Received: 11 October 2020 / Accepted: 23 November 2020 / Published online: 5 December 2020

(c) The Author(s) 2020

\begin{abstract}
Purpose To increase patient safety, ultrasound detection acupuncture (UDA) has been developed, which can detect a safe depth for acupuncturists to avoid causing pneumothorax. This study aims to develop and evaluate a single-transducer ultrasound for acupuncture (UFA) to promote UDA.

Methods Special A-mode and M-mode signals were analyzed to identify the depth of the lung. Six subjects were recruited to test the reliability and validity of UFA on GB21, LV14 and BL43 acupuncture points.

Results The result showed UFA's coefficient of variation was less than 0.2 and no difference in age, gender, and BMI of the subjects statistically, demonstrating excellent reliability. However, the content validity index of 0.51 did not meet expectations. UFA has good reliability, but it cannot reach the level of medical ultrasound. UFA uses A-mode and the seashore sign of M-mode to make a good identification of the lung, and it would be useful in the promotion of UDA.
\end{abstract}

Keywords Ultrasound $\cdot$ Acupuncture $\cdot$ Patient safety $\cdot$ Pneumothorax

JEL Classification I1 1 Analysis of Health Care Markets.

\section{Introduction}

Mark C. Hou

dr.markhou@gmail.com

Ying-Ling Chen

lingcmu@cmu.edu.tw

Shun-Chang Chang

140816@cch.org.tw

Kai-Wen Chuang

182267@cch.org.tw

Po-Yang Lee

chelseasfox@gmail.com

Chih-Chung Huang

cchuang1201@gmail.com

1 School of Traditional Chinese Medicine, China Medical University, No. 91 Xueshi Road, North District,

Taichung City 40402, Taiwan

2 Department of Chinese Medicine, Changhua Christian Hospital, 135 Nan-Siao St., Changhua 50006, Taiwan

3 Department of Biomedical Engineering, National Cheng Kung University, No. 1, Daxue Road, Tainan City 702, Taiwan
Although the scientific community has yet to form a clear and final opinion on the physiological structure of meridians and the mechanisms of acupuncture, this form of traditional Chinese medicine [1] has seen evidence-based medicine indicate approval of its effectiveness. In this way, it was confirmed as a treatment of 28 diseases and conditions, including stroke, lower-back pain, headache, and hypertension, following a 2002 World Health Organization review of randomized clinical trials [2]. In addition, large-scale clinical trials of acupuncture have suggested that it can be used for lower-back pain, knee pain, headache in Germany [3].

As with most medical interventions, acupuncture can have adverse effects, the common ones mainly being local pain, hematoma, bleeding, ecchymosis, and pneumothorax [4-7]. These mostly relate to the operator's technique and the depth of the acupuncture [8]. Some of these adverse effects can cause serious clinical problems, with pneumothrax being one of the most serious, though their incidence is very low. Thus, we need to actively prevent their occurrence. 
Although the literature covers reference depths, the depth of each acupuncture point is different and the variability between individuals can be great and the safe depth is proportional to the patient's body mass index or anthropometric data [9-13]. In order to avoid causing pneumothorax, scholars have proposed using ultrasound to measure the depth of the lung beneath the surface of the skin [14]. Ultrasound is non-invasive, convenient to obtain, and relatively simple to operate. It is most suitable in combination with clinical acupuncture. When acupuncture is performed at high-risk areas such as the skin above the lungs, ultrasound is used to detect the safe depth of the site before the appropriate-sized needles can be chosen. This can greatly reduce the risk of puncturing internal organs, improve patient safety, and increase the quality of treatment [15].

Combining acupuncture and ultrasound is known as ultrasound detection acupuncture (UDA). Taking the chest as an example, ultrasound is used to measure the distance from the skin surface to the pleura, so a needle can be chosen that will be shorter than this depth, to avoid puncturing the lung. Though UDA is in use at some medical institutions and teaching hospitals, it has not been easy to promote it to general acupuncture clinics due to the high cost of ultrasound. Therefore, it is necessary to develop of affordable way to perform ultrasound for acupuncture.

Ultrasound is mainly used to diagnose diseases and conditions, and requires a number of sets of ultrasound transmitters and receivers to present 2D images through a special array combination commonly known in practice as brightness mode (B-mode), for differential diagnosis. It is the number of these sophisticated components that makes ultrasound so expensive $[16,17]$. Nevertheless, UDA is mainly used to detect the position of the lung, and as long as the air contained in the lungs can be detected and its depth measured, multiple sets of expensive transmitters are not required in acupuncture. Rather, UDA uses one simple ultrasound transmitter to emit a single beam that detects the difference between the basic structure of body tissue and lung air by providing amplitude mode (A-mode) data, coupled with a motion mode (M-mode) scan, to confirm lung depth [18]. In this way, it is possible to design a simple ultrasound that measures the depth of the lungs in the same way as UDA. This study sets out the development an UFA and evaluates its effectiveness for UDA.

\section{Materials and Methods}

\subsection{Ultrasound for Acupuncture (UFA)}

Sound transmission speeds vary greatly between air $(331 \mathrm{~m} / \mathrm{s})$, soft tissue $(1540 \mathrm{~m} / \mathrm{s})$, and bones $(4080 \mathrm{~m} / \mathrm{s})$ in the human body. When ultrasonic waves are passed through the skin, subcutaneous soft tissues, bones and air to the lungs, different parts cause different reflections, allowing the ultrasound to detect different body structures. Especially the lower sound transmission speed in the air can make ultrasound device easily detected the position of the lungs. It is these differences that can be used to develop an ultrasound for acupuncture (UFA) device [19, 20]. A single beam ultrasonic transducer was used to detect the difference between the body tissue and lung air by using A-mode, coupled with M-mode, to confirm the safe lung depth. A transducer was used with a penetration depth greater than $100 \mathrm{~mm}$ and a center frequency of $3.75 \mathrm{MHz}$. Thus, a A306S transducer and 5072PR ultrasonic transmitter and receiver (Panametrics, Waltham, MA, USA) and a PXI-5152 digital analog converter (National Instruments, Austin, TX, USA) were used for signal conversion to the input computer for composition. We wrote a program using Labview 2017 (PXI5152, National Instruments, Austin, TX, USA) to present the A-mode and M-mode signals to create a UFA prototype for small-scale human experiments (Fig. 1).

\subsection{Validity and Reliability of UFA}

Eight subjects (three male, three female) aged 20 to 60 years gave their consent to be included in this study, with at least one of the subjects satisfying each of the following criteria: aged 20 to 40 years old, aged 40-60 years old, having a BMI of less than 18.5, having a BMI higher than 27, suffering from chronic obstructive pulmonary disease. This was to test whether UFA is affected by gender, age, weight, and lung disease. Subjects with high blood pressure, heart disease, and diabetes, and those with major injury and illness (except lung disease) were exclude. The study has passed Institutional Review Board approval (No. 181248) and Taiwan FDA review (No. 1090004050).

The ultrasound measurement depths of the UFA were compared using Leltek ultrasound (LU700C, Leltek, New Taipei, Taiwan) (Ministry of Health Medical Device System No. 006630), a newly available ultrasound that is low-priced, portable, and convenient to operate, and is similar to UFA for the purposes of the research. Testing was performed in a room at a constant temperature of 22 degrees.

GB21, BL43, and LR14 acupuncture points were selected to represent the upper, rear, and front parts of the chest, respectively (Fig. 2). GB21 was located at the midpoint of the spinous process of the seventh cervical vertebra and the acromion end of the clavicle. LR14 was located at the sixth intercostal space, inferior to the mid-clavicle line. BL43 was located at the level with the lower border of the spinous process of the fourth thoracic vertebrae. The operator marked the sites of these three points on the subject with a marker pen, and used UFA to conduct safe depth detection twice, and recorded the results. Next, Leltek was used to make 
Fig. 1 UFA system architecture diagram
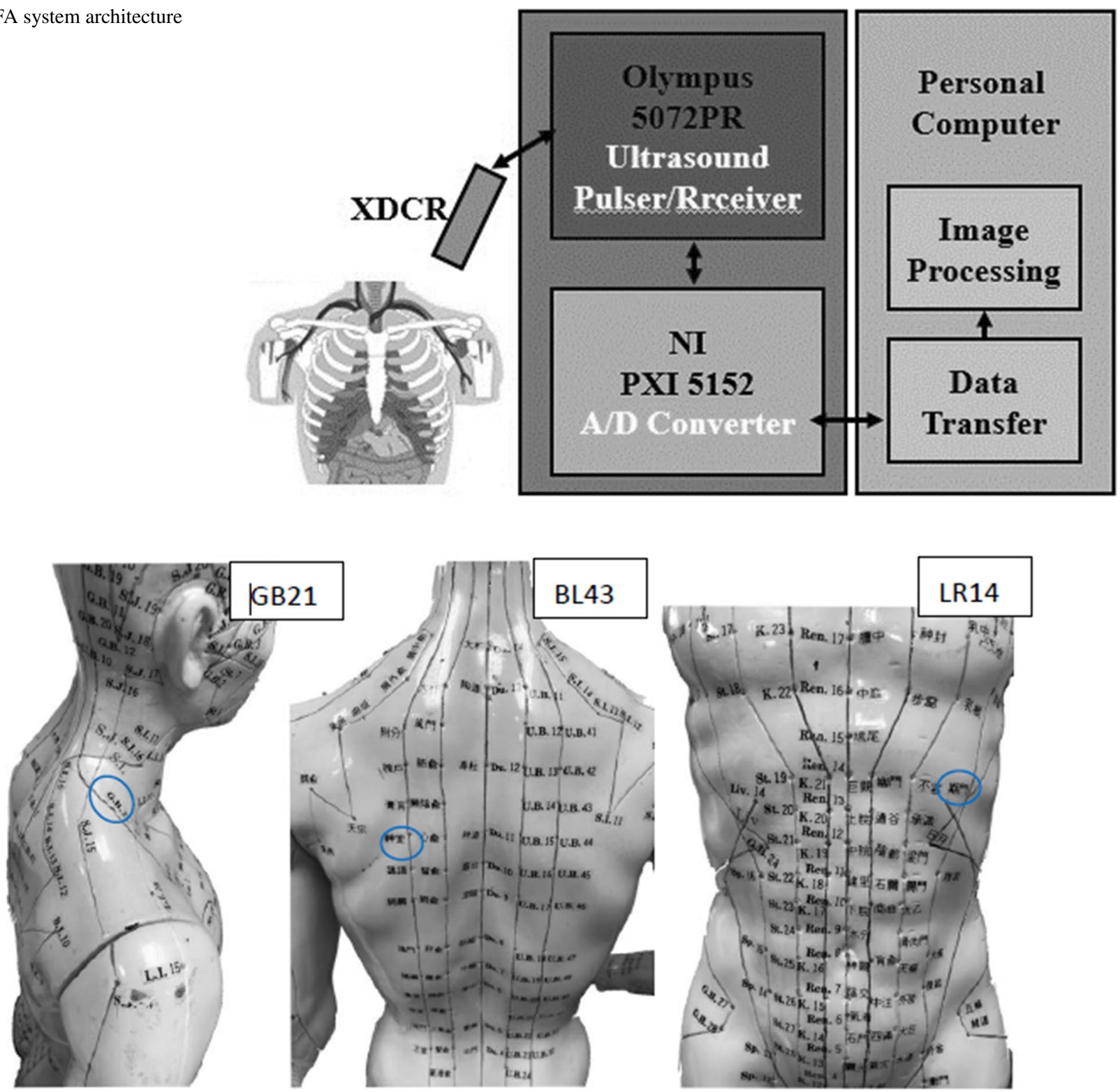

Fig. 2 Locations of CB21, BL43 and LR14. GB21, BL43, and LR14 represent the upper chest, back and front parts of the chest. (marked by circles)

the same measurements, and a photograph was printed to show the distance of the ultrasonic image. The results of the measurements of the three points were sent to five specialists with more than 7 years' experience of acupuncture individually for evaluation. A content validity index (CVI) was calculated, with an excellent CVI considered to be greater than 0.8 .

Three different tests were carried out on GB21, BL43, and LR 14 by the same operator over 20 operations, once a week for four consecutive weeks. Three operators were used at the same time to determine the robustness of the UFA [21, 22]. The average and standard deviation of the results were calculated, and the average of the standard deviation was divided into the coefficient of variation (CV). The smaller the $\mathrm{CV}$, the more concentrated the data and the higher their reliability [23]. When the CV was less than 0.2, UFA was considered to have good reliability.

The data of two subjects with same gender, age range and disease but different BMI were compared statistically. The same principle was applied to age, gender, and disease of the subjects. The Mann-Whitney U test was used to verify the reliability and validity of the values obtained. When there was no significant difference, UFA was considered not to be affected by gender, age, obesity, or lung disease state. 


\subsection{Safety of UFA}

As a non-invasive scan, ultrasound causes very little harm to human body. If used over a long period of time, it may occasionally cause swelling and discomfort in the skin surface and subcutaneous tissue [24]. Since the time taken for ultrasound measurements in this study was limited, such injuries were not expected. To protect the subjects, the skin at the GB21, BL43, and LR14 points was observed before and after the measurements were taken before and after the test to identify if there had been any impact to ensure the safety of UFA.

\section{Result}

\subsection{Designing the UFA}

UFA is a prototype medical device that uses commercial equipment as hardware and custom-written software to present A-mode and M-mode signals (Fig. 3). It has passed IRB approval (No. 181248) and TFDA review (No. 1090004050).
When the UFA detects the lungs, the A-mode will experience amplitude attenuation due to the slower speed of sound transmission in the air. In M-mode, due to the difference between the skin, soft tissue, and lung tissue, a seashore sign appears in the signal. In M-mode, due to the differences of the signal among the skin, soft tissue, and lung tissue, a seashore-like image appeared. When compared M-mode and a seashore photo, we can find sky, waves and sand are refereeing to skin, soft tissue and lung grossly. The M-mode image of skin to lung was called a seashore sign by Lichtenstein et al. [25, 26] Combined with A-mode and M-mode, the lung's depth can be detected. We also demonstrated an image of M-mode that UFA compared with commercial portable ultrasound devices (Leltek). (Fig. 4). They had a high similarity.

\subsection{Reliability and Validity tests of UFA}

A total of six subjects were recruited as UFA test subjects. Since the number of 6 subjects complied with the predetermined matching principle, the recruitment was stopped after 6 subjects. (Table 1) The CVI of UFA evaluated by five
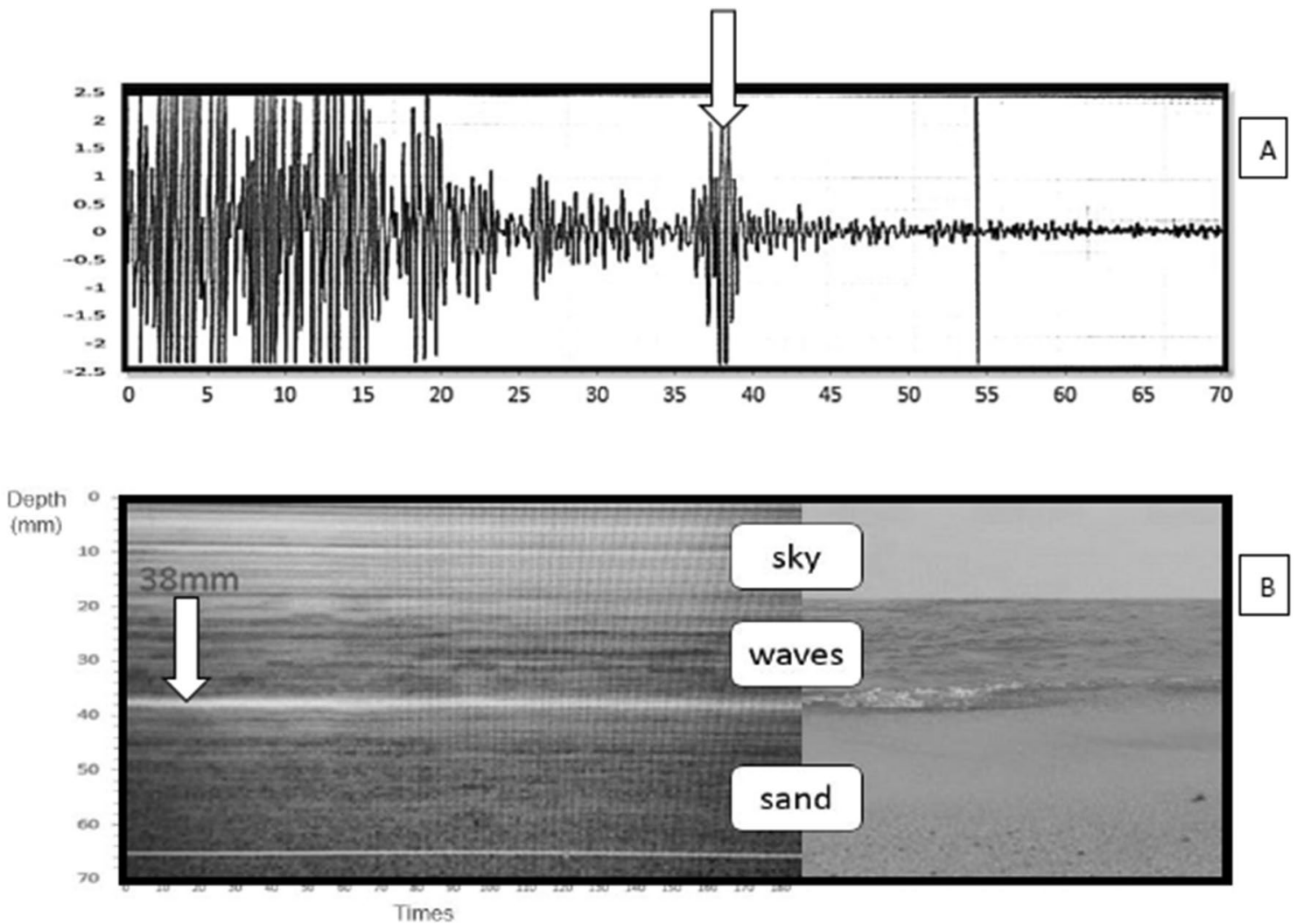

Fig. 3 A-mode and M-mode of UFA. a A-mode of UFA for detecting the depth of the lung. It showed that when the sound waves penetrated into the lung, the signal decreased greatly at about $38 \mathrm{~mm}$. This feature can be a very clear indicator for detecting of the lungs. (as indicated by the arrow) b M-mode of UFA for detecting the depth of the lung. (Left image is the M-mode, Right image is a seashore photo) The depth of the lung (sand) was $38 \mathrm{~mm}$. (as indicated by the arrow) 


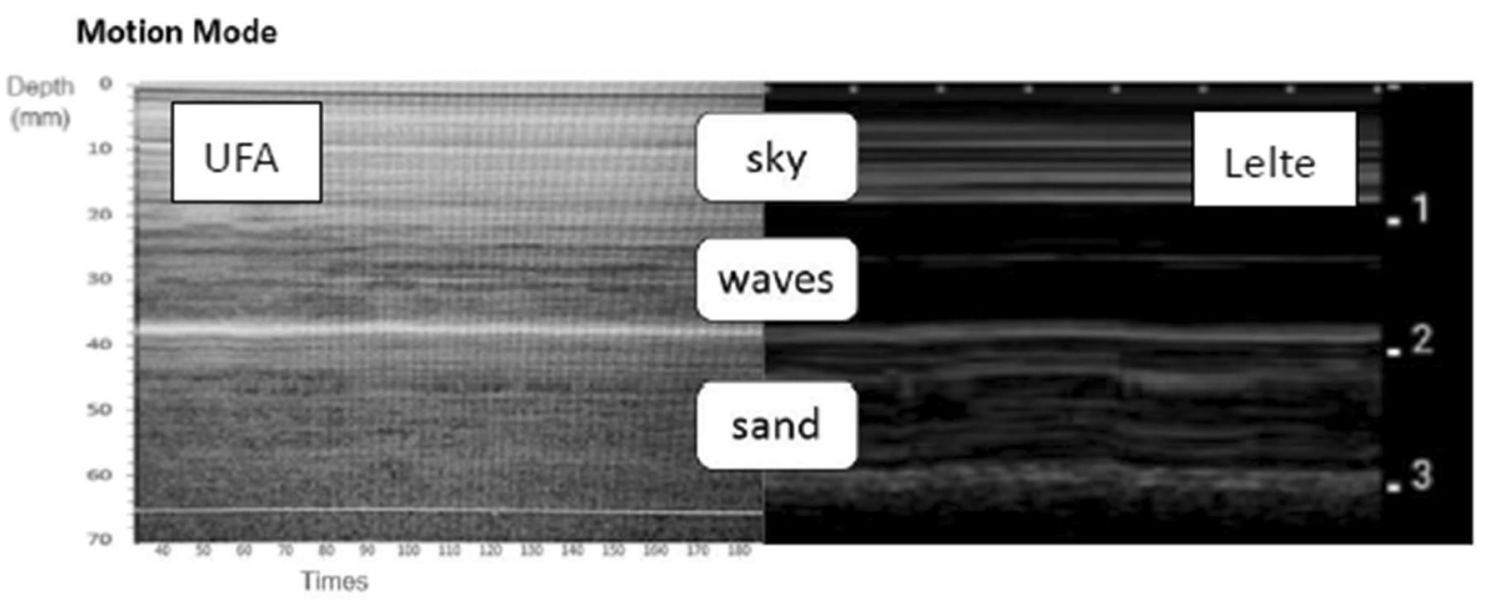

Fig. 4 M-mode of UFA and Leltek demonstrated a seashore sign

Table 1 Basic data of subjects $(\mathrm{n}=6)$

Table 2 Content Validity Index of UFA $(n=6)$

\begin{tabular}{lllllll}
\hline Subject & Gender & Age & Height & Weight & BMI & Disease \\
\hline 001 & Male & 31 & 172 & 87 & 29.4 & \\
002 & Female & 28 & 161.5 & 48.1 & 18.4 & \\
003 & Female & 29 & 161 & 58 & 22.3 & \\
004 & Male & 31 & 170 & 64 & 22.1 & \\
005 & Female & 41 & 161 & 60 & 23.1 & \\
006 & Male & 59 & 169 & 55 & 19.3 & COPD \\
\hline
\end{tabular}

\begin{tabular}{lll}
\hline & CVI & Total CVI \\
\hline GB21 & 0.53 & 0.51 \\
BL43 & 0.53 & \\
LR14 & 0.46 & \\
\hline
\end{tabular}

experts was 0.51 (Table 2), which is lower than 0.8 required to demonstrate good validity, indicating that UFA cannot achieve the effectiveness of medical ultrasound.

However, if we compare the CVI of the three acupuncture points separately, the CVI of the LR14 point (0.46) was found to be the lowest by individual CVI. It can be concluded that UFA performed poorly on the front chest and could be due to the narrow intercostal space for transmitting of ultrasound.

Reliability tests were performed on the GB21, BL43, and LR14 points. Results from different operating conditions show the same operator over 20 operations, the same operator at different times (once a week for four consecutive weeks), and three different operators operating at the same time (Table 3). All coefficients of variation are less than 0.2 . It is determined that UFA did not differ depending on the operator or operating time, and had excellent reliability.

In comparing whether UFA's stability was the same in subjects with different biological characteristics, the test results found that regardless of gender $(p=1)$, age $(p=1)$, BMI $(p .=1)$, the $p$-values were all greater than 0.05 , and there was no statistical difference, showing that stability was not affected. The subject with COPD (Chronic Obstruction Pulmonary Disease) did not receive this evaluation due to the poor health condition. The aim of this study was to develop an ultrasound for acupuncture, which could detect the depth of the lung in order to prevent pneumothorax when performing acupuncture. To validate that particular function of UFA, we need to test UFA under every possible circumstance of the lung. COPD patients have a thinner chest wall than ordinary people pathologically, thus this study need to include such condition for testing UFA. We are not comparing heath subjects with COPD subjects. In this study, the subject of COPD participated at the validity test, but only partial of reliability due to his poor respiratory function.

\subsection{Safety tests of UFA}

Local skin changes in each subject were observed before and after the test. A comparison of these photos revealed that UFA did not cause local skin damage. 
Table 3 Coefficients of variation of LR14, BL43, and GB21 for different operating conditions (no=6)

\begin{tabular}{lllllll}
\hline Subject & 001 & 002 & 003 & 004 & 005 & 006 \\
\hline LR14 & & & & & & \\
$\quad$ Three operators & 0.04 & 0.07 & 0.04 & 0.03 & 0.07 & - \\
Different dates & 0.05 & 0.12 & 0.05 & 0.04 & 0.03 & - \\
Operate repeatedly & 0.05 & 0.13 & 0.03 & 0.03 & 0.03 & 0.03 \\
BL43 & & & & & & \\
Three operators & 0.02 & 0.01 & 0.10 & 0.01 & 0.01 & - \\
Different dates & 0.04 & 0.07 & 0.06 & 0.05 & 0.04 & - \\
Operate repeatedly & 0.03 & 0.03 & 0.13 & 0.02 & 0.05 & 0.06 \\
GB21 & & & & & & \\
Three operators & 0.03 & 0.04 & 0.05 & 0.02 & 0.04 & - \\
Different dates & 0.04 & 0.05 & 0.01 & 0.05 & 0.04 & - \\
Operate repeatedly & 0.06 & 0.05 & 0.04 & 0.02 & 0.02 & 0.06 \\
\hline
\end{tabular}

Three different test situations: (1) Three operators used at the same date for 10 tunes. (2) The same operator used over 10 times once a week for four consecutive weeks. (3) The same operator repeatedly used over 20 times

\section{Discussion}

The purpose of the UFA was to design an ultrasound for acupuncturists that is affordable and which can accurately detect lung depth. It discards the common multiple-channel sound source in favor of a single channel as the transmission sound source, which can reduce costs and conform to a single-point method of clinical acupuncture. In terms of imaging, the device produces only single-point polymerization imaging in A-mode and M-mode, and not 2D images. Because M-mode can locate the lungs with a seashore sign, and A-mode has a significant signal attenuation performance for the air contained in the lungs, it is easier to locate the depth of the lungs than with the 2D B-mode. This is especially valuable for acupuncturists who lack ultrasound training. Through a combination of A-mode and M-mode, acupuncturists can quickly master the skills of detecting lung depth.

The most significant finding is that this combination of ultrasonic A-mode and M-mode was very successful at detecting lung depth. Ultrasound has traditionally used B-mode for qualitative and localization, whereby the relevant anatomy needs to be understood, and the subject is asked to breathe hard to cause the pleura to undulate, in order to confirm the position and depth of the lungs. Therefore, it is not easy to learn how to detect lung position using B-mode and it is prone to errors. On the contrary, A-mode without signal in the lungs and $\mathrm{M}$ mode to show a seashore image helps the operator to easily grasp the depth of the lungs. Although some scholars have proposed the use of coastlines in the past, they have not matched the use of A-mode [26]. We believe that UFA is very useful lung detection technology that can make it easier to identify the safe depth during acupuncture. Lin et al. conducted the earliest relevant research to analyze the safety depth of acupuncture points by gross anatomy and CT, finding that the degree of human body variation was very large and related to BMI. Two of the subjects in this study had BMI of 29.4 and 18.4, respectively. The depth differences between these two in the GB21, LV21, and BL43 points showed a similar positive linear correlation, just as Chou et al. [12] and Chu et al. found that the safety depth was not only positively correlated with BMI, but also positively correlated with other anthropometric data [27]. In addition, Chen measured the safety depth of 60 subjects with ultrasound on the GB21 point at $17.4 \mathrm{~mm}$ for males and $14.6 \mathrm{~mm}$ for females, which is far from the average depth measured in the six subjects in this study with LELTEK $(28.7 \mathrm{~mm})$ and UFA $(32.4 \mathrm{~mm})$. Clinical acupuncture GB21 operation with a one-inch needle was found not to produce pneumothorax. The body part of the one-inch needle is $25 \mathrm{~mm}$, so though Chen's result was shallow, it may be that the pleura confirmation method was different [14].

UFA showed excellent stability performance. Whether operated repeatedly by the same operator 20 times, repeated by the same operator for four weeks, or operated by three different operators over the same period, the three different test methods maintained a standard CV of less than 0.2 . It can be said that the stability of being unbroken was achieved. There was no difference in gender, age, or BMI performance, showing that UFA can accurately carry out performance tests on subjects with different biological characteristics. There was no visible damage to the skin before and after the test, which showed it is very safe. UFA's excellent reliability and security are partly due to its use of hardware components from reputable commercial manufacturers, such as Olympus. On the other hand, it was also important that we accurately analyzed the original signal and presented reliable data in A-mode and $\mathrm{M}$-mode.

Pleura is a very important anatomical marker in the measurement of UFA. In ultrasonic imaging of the lungs, pleura 
sliding is also an important marker [28]. The normal pleura thickness is between 0.2 and $0.44 \mathrm{~mm}$ [29] with a pleural space in the middle. Sometimes effusion will form and the pleura will appear thicker on the ultrasound image and show as a white stripe. This is also the basis for general medical ultrasound to identify the lungs. UFA is based on the air in the lungs covered by pleura as the measurement target; therefore, it may be a little deeper than the depth measured by medical ultrasound. We believe that this is the reason why UFA cannot achieve good validity. In the comparison between UFA and medical ultrasound, the lung depth measured by UFA is about $0.4-1.2 \mathrm{~cm}$ deeper than that of medical ultrasound, which caused the CFA of UFA to reach 0.8 after evaluation by experts.

The UFA measurement depth does not appear to be shallower than medical ultrasound, which shows that the error is systematic rather than random. We infer that a possible reason for the is that UFA provides is a single-point image measured by single-point, one-channel sound emission, which is different to the $2 \mathrm{D}$ image recovered by the medical ultrasonic multi-point 120 channel emission at the measured pleura part. If the UFA data are subtracted by $0.8 \mathrm{~cm}$ to correct, the depth of the two is not too far away. As for the true depth of the lungs, only CT, or surgery are more accurate.

With the rapid progress of technology, many low-cost, easy-to-use ultrasounds have appeared in the last year, including butterfly iQ (Butterfly Network, Inc. Guilford, CT. USA) and Leltek. The aim of this study was to design ultrasound that is affordable for general acupuncture clinicians, so we compared UFA with Leltek instead of the more expensive Sonosite model that we had originally planned to use. In this way, we could test at the same time whether these newly launched ultrasounds can also be used in clinical acupuncture. This is more beneficial for the promotion of UDA.

The biggest difference between UFA and general ultrasound is the purpose of its use. Ultrasound is mainly used to diagnose lesions, and so it is biased toward the needs of resolution and stereo imaging. UFA is mainly used to measure the depth of internal organs by identifying anatomical markers such as the pleura. Because the ultrasound target for acupuncture is simple and the positioning is clear, it is possible to provide clinically useful tools by using low-order ultrasound with easy-to-master ultrasound characteristics.

The next step will try to improve the validity of UFA. We found LR14 was the most difficult testing point using UFA. LR14 located at the front chest and at the 6th intercostal space. UFA used a Linear probe which made it hard to attach to the skin at that oblique sphere area properly. A convex probe would work better. Thus we will add a convex probe beside Linear probe to be used at the LR14 in the next version of UFA.

Point of care ultrasound uses ultrasound widely in emergency and intensive care, allowing medical staff to use ultrasonic technology across departments to improve their grasp of the disease [30, 31]. Similarly, in order to improve the quality of acupuncture and modernize technology, we have developed UDA. With good tools, acupuncturists can use the higher risk points on the chest, such as BL, GB, LV, and other important but less-used points without fear. The LR14 and BL43 measured in this study are both important acupuncture points for the treatment of the heart and lungs, but they have been gradually abandoned due to concerns over the safety of patients [8]. With new tools and techniques, new clinical guidelines can be established for acupuncture. Devising a method for clinicians to follow acupuncture points would not only improve patient safety, but also strengthen the core competence and value of TCM acupuncture.

Modern medicine is closely integrated with modern science, and new technologies, and tools are being introduced. Although acupuncture has a long history, it is very slow to take up modern technology, however. So far, electricity, infrared rays, medicine, and microwaves have been added to acupuncture. Many scholars have continued to work hard in this regard $[8,32-34]$, although more peer input is required. This research institute is willing to cooperate with the industry with UFA in the future, and doing so may add some color to acupuncture innovation.

Acknowledgements The study thanks Ms. Wei Han Chen for her assistance. We like to thanks Dr. WeiOHen Wu and Dr. Mei-Chia Hsu for their help of graphic preparation. This research is funded by the Ministry of Science and Technology (MOST 108-2511-H-371-001-MY2) and Changhua Christian Hospital (108-CCH-IRP-063).

Author Contributions All research done by the authors.

Funding Ministry of Science and Technology (MOST 108-2511-H-371-001-MY2) and Changhua Christian Hospital (108-CCH-IRP-063).

Data Availability We provided our data at google disk for reference. (https://drive.google.com/drive/folders/1uGDcTT6ejzypKqt8_XsAcY 4YNKR0bmM8?usp=sharing).

Code availability Not applicable.

\section{Compliance with Ethical Standards}

Conflict of interest The authors declares that they have no conflict of interest.

Ethical approval Institutional Review Board approval (No. 181248) and Taiwan FDA review (No. 1090004050).

Open Access This article is licensed under a Creative Commons Attribution 4.0 International License, which permits use, sharing, adaptation, distribution and reproduction in any medium or format, as long as you give appropriate credit to the original author(s) and the source, provide a link to the Creative Commons licence, and indicate if changes were made. The images or other third party material in this article are 
included in the article's Creative Commons licence, unless indicated otherwise in a credit line to the material. If material is not included in the article's Creative Commons licence and your intended use is not permitted by statutory regulation or exceeds the permitted use, you will need to obtain permission directly from the copyright holder. To view a copy of this licence, visit http://creativecommons.org/licenses/by/4.0/.

\section{References}

1. Lao, L. (2008). Acupuncture clinical studies and evidence-based medicine-an update. Zhen ci yan jiu = Acupuncture Research, 33(1), 53-61.

2. World Health Organization. (2002). Acupuncture: Review and analysis of reports on controlled clinical trials. Geneva: World Health Organization.

3. Manheimer, E., Linde, K., Lao, L., Bouter, L. M., \& Berman, B. M. (2007). Meta-analysis: Acupuncture for osteoarthritis of the knee. Annals of Internal Medicine, 146(12), 868-877.

4. Ernst, G., Strzyz, H., \& Hagmeister, H. (2003). Incidence of adverse effects during acupuncture therapy-A multicentre survey. Complementary Therapies in Medicine, 11(2), 93-97.

5. Melchart, D., Weidenhammer, W., Streng, A., Reitmayr, S., Hoppe, A., Ernst, E., \& Linde, K. (2004). Prospective investigation of adverse effects of acupuncture in 97733 patients. Archives of Internal Medicine, 164(1), 104-105.

6. Shinbara, H., Ogasawara, C., Hayama, S., Hino, K., Taniguchi, H., \& Sumiya, E. (2012). A survey of adverse events at acupuncture clinics in Japan. Journal of the Japan Society of Acupuncture and Moxibustion, 62(4), 315-325.

7. White, A. (2004). A cumulative review of the range and incidence of significant adverse events associated with acupuncture. Acupuncture in Medicine, 22(3), 122-133.

8. Kim,S., Lee, S., Ha, W.-B., Lee, J.-H., Jung, H.-J., Chu, H.-M., ... et al. (2017).Development of an ultrasound-imaging procedure and acquisition of ultrasoundimages of acupuncture points for safety and accuracy of needle insertion. IntegrativeMedicine Research, 6(4), 427-433.

9. Chen, H.-N., Lin, J.-G., Yang, A. D., \& Chang, S.-K. (2008). Safe depth of abdominal acupoints in pediatric patients. Complementary Therapies in Medicine, 16(6), 331-335.

10. Chen, H.-N., Lin, J.-G., Ying, L.-C., Huang, C.-C., \& Lin, C.-H. (2009). The therapeutic depth of abdominal acupuncture points approaches the safe depth in overweight and in older children. The Journal of Alternative and Complementary Medicine, 15(9), 1033-1037.

11. Chou, P.-C., Chu, H.-Y., \& Lin, J.-G. (2011). Safe needling depth of acupuncture points. The Journal of Alternative and Complementary Medicine, 17(3), 199-206.

12. Chou, P.-C., Huang, Y.-C., Hsueh, C.-J., Lin, J.-G., \& Chu, H.-Y. (2015). Retrospective study using MRI to measure depths of acupuncture points in neck and shoulder region. BMJ Open, 5(7), e007819.

13. Lin, J.-G., Chou, P.-C., \& Chu, H.-Y. (2013). An exploration of the needling depth in acupuncture: The safe needling depth and the needling depth of clinical efficacy. EvidenceBased Complementary and Alternative Medicine. https://doi. org/10.1155/2013/740508.

14. Chen, H.-N., Chang, C.-Y., Chen, L.-Z., Chang, Y.-J., \& Lin, J.-G. (2018). Using ultrasonography measurements to determine the depth of the GB 21 acupoint to prevent pneumothorax. Journal of Acupuncture and Meridian Studies, 11(6), 355-360.

15. Chen, Y.-L., \& Hou, M. C. (2019). Ultrasound detection acupuncture needling training: Description of the method.
Acupuncture-resolving old controversies and pointing new pathways. London: IntechOpen.

16. Bouhemad, B., Zhang, M., Lu, Q., \& Rouby, J.-J. (2007). Clinical review: Bedside lung ultrasound in critical care practice. Critical Care, 11(1), 1-9.

17. Smith, S. W., Pavy, H. G., \& von Ramm, O. T. (1991). High-speed ultrasound volumetric imaging system. I. Transducer design and beam steering. IEEE Transactions on Ultrasonics, Ferroelectrics, and Frequency Control, 38(2), 100-108.

18. Carovac, A., Smajlovic, F., \& Junuzovic, D. (2011). Application of ultrasound in medicine. Acta Informatica Medica, 19(3), 168.

19. Duck, F. (1990). Physical properties of tissue: A comprehensive reference book San Diego. California: Academic Press.

20. Mast, T. D. (2000). Empirical relationships between acoustic parameters in human soft tissues. Acoustics Research Letters Online, 1(2), 37-42.

21. Hou, M. C., Huang, S.-C., Wang, H.-M., Tseng, C.-L., Lo, L.-C., \& Chen, Y.-L. (2012). A computerized system of nail-fold capillaroscopy for dry eye disease diagnosis. Multidimensional Systems and Signal Processing, 23(4), 515-524.

22. Mugii,N., Hasegawa, M., Hamaguchi, Y., Tanaka, C., Kaji, K., Komura, K., ... et al.(2009). Reduced red blood cell velocity in nail-fold capillaries as a sensitiveand specific indicator of microcirculation injury in systemic sclerosis. Rheumatology,48(6), 696-703.

23. Reed, G. F., Lynn, F., \& Meade, B. D. (2002). Use of coefficient of variation in assessing variability of quantitative assays. Clinical and Diagnostic Laboratory Immunology, 9(6), 1235-1239.

24. Liu,Y., Zhang, W. W., He, M., Gong, C., Xie, B., Wen, X., ... Zhang, L. (2018).Adverse effect analysis of high-intensity focused ultrasound in the treatmentof benign uterine diseases. International Journal of Hyperthermia, 35(1),56-61.

25. Lichtenstein, D. A. (2014). Lung ultrasound in the critically ill. Annals of Intensive Care, 4(1), 1.

26. Ristić-An\djelkov,A., Mladenović, Z., Baškot, B., Babić, S., Ristić, M., Mišić, T., ... et al.(2015). The role of ultrasound in assessment of lung tissue, 84. https://doi.org/10.2298/VSP15 0607084R.

27. Chu,H., Kang, G., Park, S., Kim, J., Jang, J., Park, K., ... Lee, S. (2018).Determining maximal safe needling depth based on anthropometric measurements:Focusing on acupoint GB21. European Journal of Integrative Medicine, 21,11-15.

28. Lichtenstein, D. A., \& Menu, Y. (1995). A bedside ultrasound sign ruling out pneumothorax in the critically III: Lung sliding. Chest, 108(5), 1345-1348.

29. Sureka, B., Thukral, B. B., Mittal, M. K., Mittal, A., \& Sinha, M. (2013). Radiological review of pleural tumors. The Indian Journal of Radiology \& Imaging, 23(4), 313.

30. Atkinson, P., Bowra, J., Lambert, M., Lamprecht, H., Noble, V., \& Jarman, B. (2015). International Federation for Emergency Medicine point of care ultrasound curriculum. Canadian Journal of Emergency Medicine, 17(2), 161-170.

31. Zieleskiewicz,L., Muller, L., Lakhal, K., Meresse, Z., Arbelot, C., Bertrand, P.-M., ... et al.(2015). Point-of-care ultrasound in intensive care units: Assessment of 1073procedures in a multicentric, prospective, observational study. IntensiveCare Medicine, 41(9), $1638-1647$.

32. Chia, K. L., Lam, R. P. K., Lam, C. K., \& Tsui, S. H. (2018). Acupuncture in the emergency department: A systematic review of randomised controlled trials. Acupuncture in Medicine, 36(3), 183-192.

33. Litscher, G. (2009). Modernization of traditional acupuncture using multimodal computer-based high-tech methods-Recent results of blue laser and teleacupuncture from the Medical University of Graz. Journal of Acupuncture and Meridian Studies, 2(3), 202-209. 
34. Wu, J., Hu, Y., Zhu, Y., Yin, P., Litscher, G., \& Xu, S. (2015). Systematic review of adverse effects: A further step towards modernization of acupuncture in China. Evidence-Based Complementary and Alternative Medicine. https://doi.org/10.1155/2015/432467.

Publisher's Note Springer Nature remains neutral with regard to jurisdictional claims in published maps and institutional affiliations. 